\title{
Predicting Post-treatment HIV Remission: Does Size of the Viral Reservoir Matter?
}

\author{
Alexander O. Pasternak ${ }^{1 *}$, Christina K. Psomas ${ }^{2}$ and Ben Berkhout ${ }^{1}$ \\ ${ }^{1}$ Laboratory of Experimental Virology, Department of Medical Microbiology, Amsterdam UMC, University of Amsterdam, \\ Amsterdam, Netherlands, ${ }^{2}$ Department of Infectious Diseases and Internal Medicine, European Hospital, Marseille, France
}

\section{OPEN ACCESS}

Edited by:

Takeshi Yoshida

Tokyo Medical and Dental University,

Japan

Reviewed by:

Mathias Lichterfeld,

Harvard Medical School,

United States

Mohamed Abdel-Mohsen,

The Wistar Institute, United States

*Correspondence: Alexander O. Pasternak a.o.pasternak@amsterdamumc.nl; a.o.pasternak@amc.uva.n

Specialty section: This article was submitted to Virology,

a section of the journal

Frontiers in Microbiology

Received: 31 December 2020 Accepted: 08 February 2021

Published: 26 February 2021

Citation:

Pasternak AO, Psomas CK and Berkhout B (2021) Predicting Post-treatment HIV Remission: Does

Size of the Viral Reservoir Matter?

Front. Microbiol. 12:648434.

doi: 10.3389/fmicb.2021.648434
Combination antiretroviral therapy (ART) suppresses human immunodeficiency virus $(\mathrm{HIV})$ replication and improves immune function. However, due to the persistence of long-lived HIV reservoirs, therapy interruption almost inevitably leads to a fast viral rebound. A small percentage of individuals who are able to control HIV replication for extended periods after therapy interruption are of particular interest because they may represent a model of long-term HIV remission without ART. These individuals are characterized by a limited viral reservoir and low reservoir measures can predict posttreatment HIV remission. However, most individuals with a low reservoir still experience fast viral rebound. In this Perspective, we discuss the possible reasons behind this and propose to develop an integral profile, composed of viral and host biomarkers, that could allow the accurate prediction of post-treatment HIV remission. We also propose to incorporate information on the chromatin context of the proviral integration sites into the characterization of the HIV reservoir, as this likely influences the reactivation capacity of latent proviruses and, together with the actual number of intact proviruses, contributes to the replication competence of the reservoir.

Keywords: HIV, viral reservoir, antiretroviral therapy, post-treatment controllers, predictive marker, biomarker, profile

\section{INTRODUCTION}

Combination antiretroviral therapy (ART) can successfully manage human immunodeficiency virus (HIV) replication but is not curative, due to the persistence of long-lived viral reservoirs (Deeks et al., 2016; Ndung'u et al., 2019). The main reservoir is thought to reside in latently infected resting CD4 $+\mathrm{T}$ cells in peripheral blood and lymphoid tissue, although other cell types such as macrophages may contribute as well (Darcis et al., 2019; Ganor et al., 2019). Even after many years of successful treatment, ART interruption typically leads to a viral rebound within 2-4 weeks; however, some individuals, termed "post-treatment controllers," are able to control HIV replication after therapy interruption for extended periods and thus may represent a model of long-term HIV remission without ART or functional cure (Hocqueloux et al., 2010; Steingrover et al., 2010; Salgado et al., 2011; Goujard et al., 2012; Lodi et al., 2012; Van Gulck et al., 2012; Stöhr et al., 2013; Sáez-Cirión et al., 2013; Assoumou et al., 2015; Kinloch-de Loes et al., 2015; Frange et al., 2016; Maggiolo et al., 2018; Namazi et al., 2018; Violari et al., 2019). Therefore, recent years have seen accelerated research into mechanisms of HIV control in these rare individuals and in the macaque models of post-treatment control (PTC) (Strongin et al., 2020). Several excellent reviews on PTC have been published (Cockerham et al., 2016; Goulder and Deeks, 2018; Martin and Frater, 2018; 
Etemad et al., 2019), and a mathematical model of the underlying mechanisms has been proposed (Conway and Perelson, 2015). PTC is more frequent after ART initiated during early HIV infection (Namazi et al., 2018), which is not surprising given that a low viral reservoir has been consistently measured in posttreatment controllers (Goujard et al., 2012; Van Gulck et al., 2012; Sáez-Cirión et al., 2013), and early ART initiation is much more efficient in reducing the reservoir than ART initiated during chronic infection (Strain et al., 2005; Jain et al., 2013; Buzon et al., 2014). However, early ART initiation is not sufficient for PTC as most early treated individuals, even those treated very early after infection, still demonstrate fast viral rebound upon therapy interruption (Gianella et al., 2015; Colby et al., 2018). Longer ART duration has also been proposed to increase the chances of PTC (Stöhr et al., 2013; Fidler et al., 2017), but this factor on its own is insufficient to confer this phenotype. Identification of individuals with a higher probability of PTC, in whom it is safer to interrupt ART than in others, is of utmost importance in light of HIV cure research, where every therapeutic intervention necessitates an analytical treatment interruption (ATI) to assess its efficacy. A low risk of viral rebound during an ATI means low risks of reservoir replenishment, selection of drug resistance, disease progression, and HIV transmission (El-Sadr et al., 2006; Julg et al., 2019). The absence of reliable predictive markers of viral rebound complicates clinical decisionmaking on ATI and therefore hinders HIV cure research (Li et al., 2015). To fill this knowledge gap, a number of studies have been undertaken to identify biomarkers that could predict PTC or the time to viral rebound after ART interruption (Williams et al., 2014; Assoumou et al., 2015; Li et al., 2016; Sharaf et al., 2018; Pasternak et al., 2020). The latter measure may be more inclusive than the former, as PTC is a spectrum (Martin and Frater, 2018), and its definitions differ between studies, in particular in terms of the minimal duration of viral control and of the threshold for the viral rebound (Sáez-Cirión et al., 2013; Williams et al., 2014; Li et al., 2016; Namazi et al., 2018). Variable definitions of PTC also contribute to the variability in the frequency of post-treatment controllers between studies, which ranges from $<1 \%$ to $>20 \%$ and is inversely proportional to the duration of control (Hocqueloux et al., 2010; Sáez-Cirión et al., 2013; Maenza et al., 2015; Namazi et al., 2018). To standardize these definitions, Martin et al. proposed to reserve the term "PTC" for the cases of long-term continuous HIV control (several years) below the lowest possible detection limit of commercial plasma viral load assays (20 copies $/ \mathrm{mL}$ ) and to use the term "virological remission" for the intermediate cases that do not fulfill these strict criteria (Martin and Frater, 2018). Hence, using time to viral rebound as an outcome measure allows the inclusion of all these intermediate cases, increasing statistical power and potentially allowing additional insights into the mechanisms of control. On the other hand, some individuals who demonstrate various degrees of post-treatment HIV remission undergo transient viral rebound shortly after ART interruption before resuppressing the virus (Namazi et al., 2018), and measuring the time from ART interruption to the viral rebound will exclude these individuals. Clearly, improved definitions of post-treatment remission are needed to guide future HIV cure trials.

\section{Predicting Post-treatment HIV Remission: Time for a Comprehensive Approach}

A number of candidate predictive biomarkers for the virological remission have been proposed. However, the measured markers, timing of their measurement, thresholds for viral rebound, and the statistical analyses that were performed differ significantly between studies. This, in combination with limited sample sizes, resulted in different and even some contradictory conclusions. Several studies identified total HIV DNA, measured just before ART interruption, as a predictor of time to viral rebound (Goujard et al., 2012; Williams et al., 2014; Assoumou et al., 2015), and this marker even outperformed the number of intact proviruses in distinguishing individuals with post-treatment virological remission from non-controllers in a recent study (Sharaf et al., 2018). On the other hand, three independent groups reported that cell-associated (CA) HIV unspliced RNA, measured at ART interruption, could predict time to viral rebound, while total HIV DNA was not predictive in these studies (Li et al., 2016; Sneller et al., 2017; Pasternak et al., 2020). It must be noted that CA RNA was not measured in most studies that did identify total DNA as a predictor, precluding a direct comparison between these markers. Importantly, we demonstrated that the pre-treatment-interruption level of CA unspliced RNA was predictive not only of the time to viral rebound to both $>50$ and $>400$ copies/mL but also of the magnitude of the viral rebound, independently of pre-ART virological biomarkers (Pasternak et al., 2020). This suggests that measurements of the "active reservoir" (Pasternak et al., 2013) or "transcription-competent reservoir" (Baxter et al., 2018) can help support the HIV cure-directed clinical trials (Abdel-Mohsen et al., 2020). However, standardization of assays and the CA RNA transcripts that are measured is warranted in order to obtain meaningful results. Apart from the unspliced RNA, PCR-based assays have been developed to measure levels of total, completed (polyadenylated), or multiply spliced CA HIV RNA transcripts in infected individuals (Pasternak et al., 2008; Shan et al., 2013; Yukl et al., 2018). The assay for total CA RNA uses primers that bind to the HIV TAR region and thus measures the level of transcription initiation. This TAR RNA is more abundant than unspliced RNA, but most of these transcripts are short and do not encode viral proteins (Lassen et al., 2004; Yukl et al., 2018). On the other hand, the presence of multiply spliced RNA may be a more proximal surrogate of productive infection compared with unspliced RNA only (Pasternak and Berkhout, 2018). However, multiply spliced RNA is much less abundant than unspliced (Kaiser et al., 2007; Pasternak et al., 2020), due to both proviral genetic defects (as splicing requires the presence of several intact genomic regions) and latency blocks to completion of transcription and splicing (Yukl et al., 2018; Moron-Lopez et al., 2020). As a consequence, it is challenging to detect multiply spliced RNA in ART-treated individuals without ex vivo cellular stimulation, which explains why it has not yet been assessed as a potential predictor of the post-treatment remission.

In addition to CA RNA, plasma HIV RNA blips on ART were also shown to predict shorter time to rebound (Fidler et al., 2017). Further studies in larger cohorts are necessary in order 
to establish whether the total number of HIV proviruses, HIV transcriptional activity, or a combination of these markers, can be used to support HIV curative interventions. Although total HIV DNA is mostly composed of replication-defective proviruses (Bruner et al., 2016; Hiener et al., 2017), and a significant proportion of CA RNA molecules might be transcribed from such defective proviruses as well (Pollack et al., 2017; Imamichi et al., 2020), both HIV DNA and CA RNA correlate with the inducible provirus levels (Darcis et al., 2017; Cillo et al., 2018), suggesting their utility as surrogate markers of the replication-competent HIV reservoir (Avettand-Fènoël et al., 2016; Pasternak and Berkhout, 2018). In any case, it is clear that a low viral reservoir is extremely important for HIV remission, and mathematical models have been developed that are based on the assumption that the duration of remission is inversely proportional to the replication-competent HIV reservoir size (Hill et al., 2014, 2016; Conway and Perelson, 2015; Davenport et al., 2019). It would therefore seem logical that ATI performed in a group of ARTtreated individuals with low reservoir measures may result in HIV remission in a substantial proportion of cases. This has indeed been attempted by several groups (Chun et al., 2010; Calin et al., 2016; Colby et al., 2018; Pannus et al., 2020), but the absolute majority of cases experienced a quick viral rebound, suggesting that a low reservoir alone is insufficient for HIV remission and that other factors need to be considered. Here it must be noted that our understanding of the HIV reservoirs and their importance for the prediction of the posttreatment remission is still largely limited to the peripheral blood, whereas tissue reservoirs might play an even more important role. Different cellular and anatomical compartments, such as follicular $\mathrm{T}$ helper cells in the lymph node germinal centers, may serve as sanctuaries for HIV persistence under ART (Banga et al., 2016; Chaillon et al., 2020) and fuel the viral rebound upon ATI (De Scheerder et al., 2019). Although sampling peripheral blood is obviously easier, better characterization of tissue reservoirs can improve the predictive value of the HIV reservoir for the post-treatment remission.

In addition to virological markers, several host biomarkers have been proposed to predict post-treatment HIV remission. Pre-ART levels of T-cell exhaustion markers (PD-1, Tim-3, and Lag-3) have been shown to predict the time to viral rebound, although their on-ART levels were not predictive (Hurst et al., 2015). Two recent studies identified plasma and antibody glycomic biomarkers, in particular digalactosylated G2 glycans on IgG, as predictive markers of post-treatment remission (Giron et al., 2020; Offersen et al., 2020). Moreover, pre-ATI levels of HIV gp120-specific G2 glycans inversely correlated with CA HIV unspliced RNA levels (Offersen et al., 2020), providing a possible explanation why it was predictive of longer time to rebound. Although the role of cytotoxic T lymphocytes (CTLs) in post-treatment HIV remission is probably not as pronounced as in spontaneous ("elite") HIV control and post-treatment controllers mostly lack protective HLA alleles (Goujard et al., 2012; Sáez-Cirión et al., 2013; Maenza et al., 2015), this does not mean that other components of the host immunity are not important. In fact, ART initiated extremely early, during the "hyperacute" HIV infection (Fiebig stage I), rarely results in prolonged post-treatment remission (Henrich et al., 2017;
Colby et al., 2018), which is thought to reflect an insufficient time window for maturation of the adaptive immune responses (Goulder and Deeks, 2018). In contrast, the SPARTAC and Primo-SHM studies where temporary ART was started during primary infection, but not too early, resulted in some participants experiencing various degrees of post-treatment remission (Stöhr et al., 2013; Pasternak et al., 2020).

It therefore appears useful to develop a comprehensive molecular profile, incorporating multiple viral and host biomarkers, that could reliably predict post-treatment HIV remission. Such a profile could be based on the principle of diagnostic multivariate index assays that are already used in other medical fields (Zhang, 2012). The advantage of such a composite molecular profile, compared to single biomarker assays, is that the aggregated information from complementary biomarkers is expected to outperform each of the individual component biomarkers in sensitivity, specificity, and predictive value. Applied to the prediction of post-treatment HIV remission, such a profile may be composed of metabolomic, lipidomic, and proteomic biomarkers, in combination with virological and immunological profiling. In addition, the expression of recently identified cellular markers of the HIV reservoirs, such as CD32a, CD30, CD20, PD-1, and others (Fromentin et al., 2016; Descours et al., 2017; Abdel-Mohsen et al., 2018; Hogan et al., 2018; Serra-Peinado et al., 2019; Darcis et al., 2020; Neidleman et al., 2020; Adams et al., 2021), as well as T-cell phenotypic markers (Hiener et al., 2017), could be incorporated in this profile. Indeed, CD30+ CD4+ T cells, as well as expression of some HIV restriction factors, were shown to increase before viral rebound after ATI (De Scheerder et al., 2020; Prator et al., 2020). Furthermore, Mitchell et al. (2020) recently demonstrated that plasmacytoid dendritic cells can sense HIV replication before detectable viremia following treatment interruption, which was evidenced by a transient loss of IFN $\alpha$ production. Expression of cellular factors that are involved in long-term cell survival and proliferation vs. apoptosis could also play a role (Kuo et al., 2018; Angin et al., 2019). In this regard, as no single molecule has yet been described that marks all reservoir cells, a combinatorial approach will again be beneficial and perhaps even necessary. Ideally, the evolution of such a comprehensive molecular profile could allow the development of a personalized approach to HIV curative interventions. In particular, a gender-specific approach might be necessary, since several (but not all) studies demonstrated lower CA HIV RNA levels in women compared to men (Scully et al., 2019; Falcinelli et al., 2020; Gianella et al., 2020), and estrogen has been shown to repress HIV transcription (Das et al., 2018). Such approach should also include the personal medical history of each individual, namely the level of persistent immune activation despite ART, the history of comorbidities that is often associated with chronic inflammation, as well as current and historical ART regimens, all of which may contribute to the probability and timing of viral rebound.

\section{Post-treatment Remission and the HIV Reservoir Size: Are We Measuring the Right Markers?}

Low HIV reservoir is necessary but apparently not sufficient for post-treatment remission, as even individuals with very low levels 
of reservoir markers experience fast viral rebound upon ART interruption. As discussed above, one possible solution to this problem is to identify other, complementary biomarkers, thus increasing the predictive power of the resulting profile. However, another possibility is that our current toolkit simply does not allow sufficiently accurate measurement of the HIV reservoir size. The latter is defined as the number of cells carrying replicationcompetent proviruses, in other words integrated viral genomes capable of reigniting viral spread upon ART interruption (Eisele and Siliciano, 2012; Pasternak and Berkhout, 2016). However, it is difficult to estimate the real HIV reservoir size, as PCRbased methods that measure HIV DNA and RNA overestimate the reservoir because most of proviruses are genetically defective (Bruner et al., 2016). On the other hand, the quantitative viral outgrowth assay (qVOA) will not score defective proviruses, but is thought to underestimate the reservoir as only a small fraction of genetically intact proviruses can be activated ex vivo (Ho et al., 2013; Bruner et al., 2016; Kwon et al., 2020; Martin et al., 2020). The most accurate surrogate marker of the reservoir size is currently considered to be the number of intact proviruses, estimated by either full-length proviral sequencing (Bruner et al., 2016; Hiener et al., 2017; Pinzone et al., 2019) or the recently developed digital droplet PCRbased intact proviral DNA assay (IPDA) (Bruner et al., 2019). Not all intact proviruses are replication-competent, as fulllength sequencing is only able to identify gross genetic defects, such as large internal deletions, hypermutation, stop codons, frameshift mutations, or defects in the major splice donor site or the packaging signal, and will not identify other genetic changes that may be deleterious for HIV replication. However, most of the intact proviruses demonstrate normal replication kinetics in vitro (Ho et al., 2013), suggesting that the majority of proviruses identified as intact by full-length proviral sequencing are replication-competent. In comparison, IPDA overestimates the intact reservoir somewhat, as only $\sim 70 \%$ of proviruses that are identified as intact by IPDA are also intact by full-length proviral sequencing (Bruner et al., 2019).

There might be, however, another level of complexity to the measurement of HIV reservoir. By applying the novel multiple displacement amplification (MDA)-based matched integration site and proviral sequencing (MIPSeq) technique, the Lichterfeld group demonstrated that in individuals on prolonged ART, in comparison to defective proviruses, intact HIV proviruses were enriched for non-genic chromosomal positions and other features of "deep latency" (Einkauf et al., 2019). This bias was subsequently confirmed by another group that also used the MDA method (Patro et al., 2019). More importantly, the same technique, recently applied to the characterization of the HIV reservoir in elite controllers, revealed that this population demonstrates an even more extreme phenotype than ART-treated individuals: $40 \%$ of intact proviral clones in elite controllers were integrated into non-genic or pseudogenic regions, compared to $13 \%$ in ART-treated individuals (Jiang et al., 2020). Moreover, in contrast to ART-treated individuals, intact proviral sequences from elite controllers were preferentially integrated in centromeric satellite DNA or in other regions associated with heterochromatin, and at an increased distance to transcriptional start sites and accessible chromatin, and were enriched in repressive chromatin marks. As infection of CD4+ $\mathrm{T}$ cells from elite controllers ex vivo with a laboratory HIV strain led to a normal integration pattern, it is likely that this skewed integration pattern observed in vivo is the result of selective elimination of cells infected with transcriptionally competent intact proviruses over time by the immune system, resulting in enrichment for intact proviruses that are in a state of "deep latency" (also referred to as "blocked and locked" state) and are unlikely to be reactivated (Jiang et al., 2020). It was demonstrated 20 years ago that the provirus transcriptional activity is influenced by the integration site (Jordan et al., 2001), and indeed, levels of HIV transcription in elite controllers were shown to be at least 10-fold lower than in ART-treated individuals (Jiang et al., 2020), confirming the results of previous studies (Van Gulck et al., 2012; Hatano et al., 2013). Interestingly, Battivelli et al. (2018) found that HIV reactivation in a primary CD4+ T-cell model of latency occurred in at most $5 \%$ of the infected cells and depended on integration in an open chromatin context, which was confirmed by another group that demonstrated that inactive chromatin marks accumulate across the provirus with time (Lindqvist et al., 2020). The proportions of clonally expanded intact proviruses were shown to be larger in elite controllers than in ART-treated individuals (Veenhuis et al., 2018; Jiang et al., 2020), with the same pattern observed in a post-treatment controller (Veenhuis et al., 2018). Moreover, CD8 + T cells from the elite and post-treatment controllers were capable of suppressing replication of their autologous clonally expanded viruses in vitro (Veenhuis et al., 2018), suggesting that these intact proviruses can undergo clonal expansion without or with minimal viral gene expression (Hosmane et al., 2017; Musick et al., 2019).

Taken together, these recent insights imply that the integration site-imposed reactivation potential of a provirus could be as important as its genetic intactness (Chomont, 2020). In other words, not only the size of the reservoir, but also its repertoire (not only in terms of chromatin context but also in terms of diversity and clonality of proviral integration sites) matters for the replication competence. Moreover, they bring into doubt the concept that qVOA profoundly underestimates the replication-competent reservoir, providing a possible explanation why only a tiny fraction of intact proviruses can be reactivated ex vivo. On the other hand, Ho et al. reported that most non-induced intact proviruses in their study were integrated into active transcription units, suggesting that other factors exist that prevent intact provirus reactivation, at least ex vivo (Ho et al., 2013). Although the reactivation abilities ex vivo and in vivo cannot be directly compared, and there always is a possibility that a provirus that cannot become reactivated ex vivo even after multiple rounds of TCR stimulation, still can reignite viral rebound in vivo after ART interruption, we might consider the number of intact proviruses as the upper, conservative, limit of the replication-competent reservoir. In most infected individuals, the reservoir is probably much lower than this limit and in order to be able to accurately quantify the reservoir size, it would be necessary to combine the measurement of genetic intactness with that of in vivo 


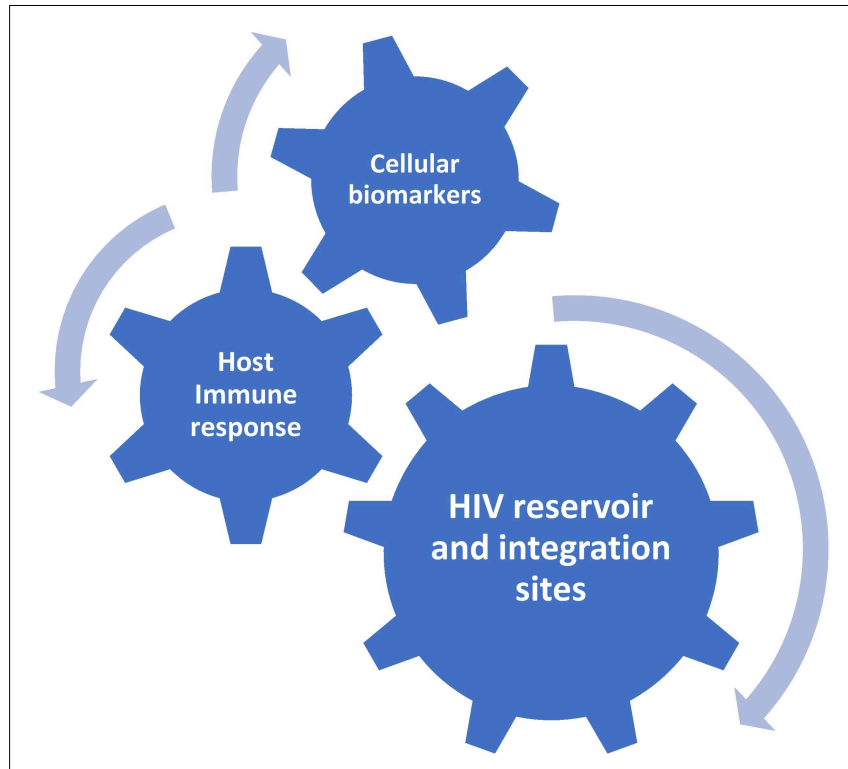

FIGURE 1 | Proposed molecular profile for the prediction of post-treatment HIV remission.

reactivation potential, although measuring the latter is very difficult if not impossible. As a surrogate, a simple score based on the provirus intactness and the chromosomal context of its integration site could be developed, as MDA-based and other similar assays that simultaneously measure the provirus intactness and map the integration site provide the possibility to do this. Importantly, such a combined score may be able to predict time to viral rebound after ATI and/or post-treatment HIV remission better than other reservoir measures. To further improve the predictive power, this score could be incorporated into the molecular profile proposed above (Figure 1). Indeed, although the mechanisms of control are different between most elite and post-treatment controllers, studies have identified a subpopulation of elite controllers with both markedly inefficient ex vivo $\mathrm{HIV}$ reactivation from resting $\mathrm{CD} 4+\mathrm{T}$ cells and low HIV-specific CD8+ T-cell responses (Noel et al., 2016; Canouï et al., 2017). This subpopulation may in fact resemble posttreatment controllers, in most of which no protective HLA alleles were found and CD8+ responses are also not particularly strong, and the virus replication is probably controlled due to infrequent reactivation from latency. Further research is needed to establish whether the HIV integration site landscape in posttreatment controllers resembles that of elite controllers (Jiang et al., 2020). This should involve longitudinal studies to evaluate immune selection for viral reservoir cells (Wang et al., 2018; Huang et al., 2019).

Finally, if the proviral integration sites are so important for the replication competence of the reservoir, is there something that can be done therapeutically to facilitate HIV integration in regions that are associated with the repression of transcription? HIV preferentially integrates in actively transcribed genes (Han et al., 2004; Marini et al., 2015) but long-term ART selects for transcriptionally silent proviruses (Pinzone et al., 2019).
However, ART cannot do wonders and even after decennia of suppressive therapy, most individuals will experience a fast viral rebound upon ART interruption. Therefore, a number of strategies to "block and lock" the provirus in the inactive state are currently under investigation (reviewed in Moranguinho and Valente, 2020; Vansant et al., 2020a). In particular, the Debyser group developed a technique, based on the smallmolecule (LEDGIN) inhibition of the interaction between the HIV integrase and its host cofactor LEDGF/p75, that allows retargeting HIV integration from active genes to sites that are less transcriptionally active, indeed resulting in lower HIV transcription (Vranckx et al., 2016; Vansant et al., 2020b). However, it is still unclear how this technique could be applied in infected individuals, as the HIV reservoir is formed very early after infection and once the provirus is integrated, it cannot be retargeted. Interestingly, several groups recently reported that in the untreated infection, the reservoir turns over quickly, and that most proviruses in ART-treated individuals match circulating HIV variants from shortly before ART initiation (Brodin et al., 2016; Abrahams et al., 2019; Pankau et al., 2020). In this case, treatment with LEDGINs or similar compounds shortly before the start of ART could indeed result in a lower transcriptional activity of the reservoir and, as a consequence, a higher frequency of post-treatment HIV remission.

\section{CONCLUSION}

In summary, although a number of biomarkers are already identified that can predict post-treatment HIV remission, there are still major gaps in our understanding of its underlying mechanisms. Consequently, ATIs are still the only way to assess the efficacy of new HIV curative interventions, and criteria for the recruitment of clinical trial participants remain unclear. Further research is urgently needed to identify robust and validated predictive biomarkers of post-treatment remission. In this regard, the development of an integral biomarker profile as outlined above should facilitate the efforts to achieve prolonged virological control in the absence of ART.

\section{DATA AVAILABILITY STATEMENT}

The original contributions presented in the study are included in the article/Supplementary Material, further inquiries can be directed to the corresponding author/s.

\section{AUTHOR CONTRIBUTIONS}

AP wrote the first draft of the manuscript. CP contributed to the figure. All authors contributed to the writing and approved the final manuscript.

\section{FUNDING}

AP is supported by the grant no. 09120011910035 from the Dutch Medical Research Council (ZonMw). 


\section{REFERENCES}

Abdel-Mohsen, M., Kuri-Cervantes, L., Grau-Exposito, J., Spivak, A. M., Nell, R. A., Tomescu, C., et al. (2018). CD32 is expressed on cells with transcriptionally active HIV but does not enrich for HIV DNA in resting T cells. Sci. Transl. Med. 10:eaar6759. doi: 10.1126/scitranslmed.aar6759

Abdel-Mohsen, M., Richman, D., Siliciano, R. F., Nussenzweig, M. C., Howell, B. J., Martinez-Picado, J., et al. (2020). Recommendations for measuring HIV reservoir size in cure-directed clinical trials. Nat. Med. 26, 1339-1350. doi: 10.1038/s41591-020-1022-1

Abrahams, M. R., Joseph, S. B., Garrett, N., Tyers, L., Moeser, M., Archin, N., et al. (2019). The replication-competent HIV-1 latent reservoir is primarily established near the time of therapy initiation. Sci. Transl. Med. 11:eaaw5589. doi: 10.1126/scitranslmed.aaw5589

Adams, P., Fievez, V., Schober, R., Amand, M., Iserentant, G., Rutsaert, S., et al. (2021). CD32+ CD4+ memory T cells are enriched for total HIV-1 DNA in tissues from humanized mice. iScience 24:101881. doi: 10.1016/j.isci.2020. 101881

Angin, M., Volant, S., Passaes, C., Lecuroux, C., Monceaux, V., Dillies, M. A., et al. (2019). Metabolic plasticity of HIV-specific CD8+ T cells is associated with enhanced antiviral potential and natural control of HIV-1 infection. Nat. Metab. 1, 704-716. doi: 10.1038/s42255-019-0081-4

Assoumou, L., Weiss, L., Piketty, C., Burgard, M., Melard, A., Girard, P. M., et al. (2015). A low HIV-DNA level in peripheral blood mononuclear cells at antiretroviral treatment interruption predicts a higher probability of maintaining viral control. AIDS 29, 2003-2007. doi: 10.1097/qad. 0000000000000734

Avettand-Fènoël, V., Hocqueloux, L., Ghosn, J., Cheret, A., Frange, P., Melard, A., et al. (2016). Total HIV-1 DNA, a marker of viral reservoir dynamics with clinical implications. Clin. Microbiol. Rev. 29, 859-880. doi: 10.1128/cmr. 00015- 16

Banga, R., Procopio, F. A., Noto, A., Pollakis, G., Cavassini, M., Ohmiti, K., et al. (2016). PD-1(+) and follicular helper T cells are responsible for persistent HIV-1 transcription in treated aviremic individuals. Nat. Med. 22, 754-761. doi: $10.1038 / \mathrm{nm} .4113$

Battivelli, E., Dahabieh, M. S., Abdel-Mohsen, M., Svensson, J. P., Tojal Da Silva, I., Cohn, L. B., et al. (2018). Distinct chromatin functional states correlate with HIV latency reactivation in infected primary CD4+ T cells. Elife 7:e3465.

Baxter, A. E., O’Doherty, U., and Kaufmann, D. E. (2018). Beyond the replicationcompetent HIV reservoir: transcription and translation-competent reservoirs. Retrovirology 15:18.

Brodin, J., Zanini, F., Thebo, L., Lanz, C., Bratt, G., Neher, R. A., et al. (2016). Establishment and stability of the latent HIV-1 DNA reservoir. Elife. 5:e18889.

Bruner, K. M., Murray, A. J., Pollack, R. A., Soliman, M. G., Laskey, S. B., Capoferri, A. A., et al. (2016). Defective proviruses rapidly accumulate during acute HIV-1 infection. Nat. Med. 22, 1043-1049. doi: 10.1038/nm.4156

Bruner, K. M., Wang, Z., Simonetti, F. R., Bender, A. M., Kwon, K. J., Sengupta, S., et al. (2019). A quantitative approach for measuring the reservoir of latent HIV-1 proviruses. Nature 566, 120-125. doi: 10.1038/s41586-0190898-8

Buzon, M. J., Martin-Gayo, E., Pereyra, F., Ouyang, Z., Sun, H., Li, J. Z., et al. (2014). Long-term antiretroviral treatment initiated at primary HIV-1 infection affects the size, composition, and decay kinetics of the reservoir of HIV-1-infected CD4 T cells. J. Virol. 88:10056-65.

Calin, R., Hamimi, C., Lambert-Niclot, S., Carcelain, G., Bellet, J., Assoumou, L., et al. (2016). Treatment interruption in chronically HIV-infected patients with an ultralow HIV reservoir. AIDS 30, 761-769. doi: 10.1097/qad. 0000000000000987

Canouï, E., Lécuroux, C., Avettand-Fenoël, V., Gousset, M., Rouzioux, C., SaezCirion, A., et al. (2017). A subset of extreme human immunodeficiency virus (HIV) controllers is characterized by a small HIV blood reservoir and a weak T-cell activation level. Open Forum Infect. Dis. 4:ofx064.

Chaillon, A., Gianella, S., Dellicour, S., Rawlings, S. A., Schlub, T. E., De Oliveira, M. F., et al. (2020). HIV persists throughout deep tissues with repopulation from multiple anatomical sources. J. Clin. Invest. 130, 1699-1712. doi: 10.1172/ jci134815

Chomont, N. (2020). HIV enters deep sleep in people who naturally control the virus. Nature 585, 190-191. doi: 10.1038/d41586-020-02438-7
Chun, T. W., Justement, J. S., Murray, D., Hallahan, C. W., Maenza, J., Collier, A. C., et al. (2010). Rebound of plasma viremia following cessation of antiretroviral therapy despite profoundly low levels of HIV reservoir: implications for eradication. AIDS 24, 2803-2808. doi: 10.1097/qad.0b013e328340a239

Cillo, A. R., Hong, F., Tsai, A., Irrinki, A., Kaur, J., Sloan, D. D., et al. (2018). Blood biomarkers of expressed and inducible HIV-1. AIDS 32, 699-708. doi: 10.1097/qad.0000000000001748

Cockerham, L. R., Hatano, H., and Deeks, S. G. (2016). Post-treatment controllers: role in HIV "Cure". research. Curr. HIV/AIDS Rep. 13, 1-9. doi: 10.1007/ s11904-016-0296-x

Colby, D. J., Trautmann, L., Pinyakorn, S., Leyre, L., Pagliuzza, A., Kroon, E., et al. (2018). Rapid HIV RNA rebound after antiretroviral treatment interruption in persons durably suppressed in Fiebig I acute HIV infection. Nat. Med. 24, 923-926. doi: 10.1038/s41591-018-0026-6

Conway, J. M., and Perelson, A. S. (2015). Post-treatment control of HIV infection. Proc. Natl. Acad. Sci. U.S.A. 112, 5467-5472. doi: 10.1073/pnas.1419162112

Darcis, G., Berkhout, B., and Pasternak, A. O. (2019). The quest for cellular markers of HIV reservoirs: any color you like. Front. Immunol. 10:2251. doi: 10.3389/ fimmu.2019.02251

Darcis, G., Bouchat, S., Kula, A., Van Driessche, B., Delacourt, N., Vanhulle, C., et al. (2017). Reactivation capacity by latency-reversing agents ex vivo correlates with the size of the HIV-1 reservoir. AIDS 31, 181-189. doi: 10.1097/qad. 0000000000001290

Darcis, G., Kootstra, N. A., Hooibrink, B., van Montfort, T., Maurer, I., Groen, K., et al. (2020). CD32+ CD4+ T cells are highly enriched for HIV DNA and can support transcriptional latency. Cell Rep. 30, 2284-2296.e3.

Das, B., Dobrowolski, C., Luttge, B., Valadkhan, S., Chomont, N., Johnston, R., et al. (2018). Estrogen receptor-1 is a key regulator of HIV-1 latency that imparts gender-specific restrictions on the latent reservoir. Proc. Natl. Acad. Sci. U.S.A. 115, E7795-E7804.

Davenport, M. P., Khoury, D. S., Cromer, D., Lewin, S. R., Kelleher, A. D., and Kent, S. J. (2019). Functional cure of HIV: the scale of the challenge. Nat. Rev. Immunol. 19, 45-54. doi: 10.1038/s41577-018-0085-4

De Scheerder, M. A., Van Hecke, C., Zetterberg, H., Fuchs, D., De Langhe, N., Rutsaert, S., et al. (2020). Evaluating predictive markers for viral rebound and safety assessment in blood and lumbar fluid during HIV-1 treatment interruption. J. Antimicrob. Chemother. 75, 1311-1320. doi: 10.1093/jac/ dkaa003

De Scheerder, M. A., Vrancken, B., Dellicour, S., Schlub, T., Lee, E., Shao, W., et al. (2019). HIV rebound is predominantly fueled by genetically identical viral expansions from diverse reservoirs. Cell Host Microbe 26, 347-358.e7.

Deeks, S. G., Lewin, S. R., Ross, A. L., Ananworanich, J., Benkirane, M., Cannon, P., et al. (2016). International AIDS society global scientific strategy: towards an HIV cure 2016. Nat. Med. 22, 839-850.

Descours, B., Petitjean, G., López-Zaragoza, J. L., Bruel, T., Raffel, R., Psomas, C., et al. (2017). CD32a is a marker of a CD4 T-cell HIV reservoir harbouring replication-competent proviruses. Nature 543, 564-567. doi: 10. 1038/nature21710

Einkauf, K. B., Lee, G. Q., Gao, C., Sharaf, R., Sun, X., Hua, S., et al. (2019). Intact HIV-1 proviruses accumulate at distinct chromosomal positions during prolonged antiretroviral therapy. J. Clin. Invest. 129, 988-998. doi: 10.1172/ jci124291

Eisele, E., and Siliciano, R. F. (2012). Redefining the viral reservoirs that prevent HIV-1 eradication. Immunity 37, 377-388. doi: 10.1016/j.immuni.2012.08.010

El-Sadr, W. M., Lundgren, J., Neaton, J. D., Gordin, F., Abrams, D., Arduino, R. C., et al. (2006). CD4+ count-guided interruption of antiretroviral treatment. N. Engl. J. Med. 355, 2283-2296. doi: 10.1056/nejmoa062360

Etemad, B., Esmaeilzadeh, E., and Li, J. Z. (2019). Learning from the exceptions: HIV remission in post-treatment controllers. Front. Immunol. 10:1749. doi: 10.3389/fimmu.2019.01749

Falcinelli, S. D., Shook-Sa, B. E., Dewey, M. G., Sridhar, S., Read, J., Kirchherr, J., et al. (2020). Impact of biological sex on immune activation and frequency of the latent HIV reservoir during suppressive antiretroviral therapy. J. Infect. Dis. 222, 1843-1852. doi: 10.1093/infdis/jiaa298

Fidler, S., Olson, A. D., Bucher, H. C., Fox, J., Thornhill, J., Morrison, C., et al. (2017). Virological blips and predictors of post treatment viral control after stopping ART started in primary HIV infection. J. Acquir. Immune Defic. Syndr. 74, 126-133. doi: 10.1097/qai.0000000000001220 
Frange, P., Faye, A., Avettand-Fenoël, V., Bellaton, E., Descamps, D., Angin, M., et al. (2016). HIV-1 virological remission lasting more than 12 years after interruption of early antiretroviral therapy in a perinatally infected teenager enrolled in the French ANRS EPF-CO10 paediatric cohort: a case report. Lancet HIV 3:e49-e54.

Fromentin, R., Bakeman, W., Lawani, M. B., Khoury, G., Hartogensis, W., DaFonseca, S., et al. (2016). CD4+ T cells expressing PD-1, TIGIT and LAG3 contribute to HIV persistence during ART. PLoS Pathog. 12:e1005761. doi: 10.1371/journal.ppat.1005761

Ganor, Y., Real, F., Sennepin, A., Dutertre, C. A., Prevedel, L., Xu, L., et al. (2019). HIV-1 reservoirs in urethral macrophages of patients under suppressive antiretroviral therapy. Nat. Microbiol. 4, 633-644. doi: 10.1038/s41564-0180335-z

Gianella, S., Anderson, C. M., Richman, D. D., Smith, D. M., and Little, S. J. (2015). No evidence of posttreatment control after early initiation of antiretroviral therapy. AIDS 29, 2093-2097. doi: 10.1097/qad.0000000000000816

Gianella, S., Tran, S. M., Morris, S., Vargas, M., Porrachia, M., Oliveira, M. F., et al. (2020). Sex differences in CMV replication and HIV persistence during suppressive ART. Open Forum Infect. Dis. 7:ofaa289.

Giron, L. B., Papasavvas, E., Azzoni, L., Yin, X., Anzurez, A., Damra, M., et al. (2020). Plasma and antibody glycomic biomarkers of time to HIV rebound and viral setpoint. AIDS 34, 681-686. doi: 10.1097/qad.0000000000002476

Goujard, C., Girault, I., Rouzioux, C., Lécuroux, C., Deveau, C., Chaix, M. L., et al. (2012). HIV-1 control after transient antiretroviral treatment initiated in primary infection: role of patient characteristics and effect of therapy. Antivir. Ther. 17, 1001-1009. doi: 10.3851/imp2273

Goulder, P., and Deeks, S. G. (2018). HIV control: is getting there the same as staying there? PLoS Pathog. 14:e1007222. doi: 10.1371/journal.ppat.1007222

Han, Y., Lassen, K., Monie, D., Sedaghat, A. R., Shimoji, S., Liu, X., et al. (2004). Resting CD4+ T cells from human immunodeficiency virus type 1 (HIV-1)infected individuals carry integrated HIV-1 genomes within actively transcribed host genes. J. Virol. 78, 6122-6133. doi: 10.1128/jvi.78.12.6122-6133.2004

Hatano, H., Somsouk, M., Sinclair, E., Harvill, K., Gilman, L., Cohen, M., et al. (2013). Comparison of HIV DNA and RNA in gut-associated lymphoid tissue of HIV-infected controllers and noncontrollers. AIDS 27, 2255-2260. doi: 10.1097/qad.0b013e328362692f

Henrich, T. J., Hatano, H., Bacon, O., Hogan, L. E., Rutishauser, R., Hill, A., et al. (2017). HIV-1 persistence following extremely early initiation of antiretroviral therapy (ART) during acute HIV-1 infection: an observational study. PLoS Med. 14:e1002417. doi: 10.1371/journal.pmed.1002417

Hiener, B., Horsburgh, B. A., Eden, J. S., Barton, K., Schlub, T. E., Lee, E., et al. (2017). Identification of genetically intact HIV-1 proviruses in specific CD4+ T cells from effectively treated participants. Cell Rep. 21, 813-822. doi: 10.1016/j. celrep.2017.09.081

Hill, A. L., Rosenbloom, D. I., Fu, F., Nowak, M. A., and Siliciano, R. F. (2014). Predicting the outcomes of treatment to eradicate the latent reservoir for HIV-1. Proc. Natl. Acad. Sci. U.S.A. 111, 13475-13480. doi: 10.1073/pnas.1406663111

Hill, A. L., Rosenbloom, D. I., Goldstein, E., Hanhauser, E., Kuritzkes, D. R., Siliciano, R. F., et al. (2016). Real-time predictions of reservoir size and rebound time during antiretroviral therapy interruption trials for HIV. PLoS Pathog. 12:e1005535. doi: 10.1371/journal.ppat.1005535

Ho, Y. C., Shan, L., Hosmane, N. N., Wang, J., Laskey, S. B., Rosenbloom, D. I., et al. (2013). Replication-competent noninduced proviruses in the latent reservoir increase barrier to HIV-1 cure. Cell 155, 540-551. doi: 10.1016/j.cell.2013.09. 020

Hocqueloux, L., Prazuck, T., Avettand-Fenoel, V., Lafeuillade, A., Cardon, B., Viard, J. P., et al. (2010). Long-term immunovirologic control following antiretroviral therapy interruption in patients treated at the time of primary HIV-1 infection. AIDS 24, 1598-1601. doi: 10.1097/qad.0b013e32833 b61ba

Hogan, L. E., Vasquez, J., Hobbs, K. S., Hanhauser, E., Aguilar-Rodriguez, B., Hussien, R., et al. (2018). Increased HIV-1 transcriptional activity and infectious burden in peripheral blood and gut-associated CD4+ T cells expressing CD30. PLoS Pathog. 14:e1006856. doi: 10.1371/journal.ppat.1006856

Hosmane, N. N., Kwon, K. J., Bruner, K. M., Capoferri, A. A., Beg, S., Rosenbloom, D. I., et al. (2017). Proliferation of latently infected CD4+ T cells carrying replication-competent HIV-1: potential role in latent reservoir dynamics. J. Exp. Med. 214, 959-972. doi: 10.1084/jem.20170193
Huang, S. H., McCann, C. D., Mota, T. M., Wang, C., Lipkin, S. M., and Jones, R. B. (2019). Have cells harboring the HIV reservoir been immunoedited? Front. Immunol. 10:1842. doi: 10.3389/fimmu.2019.01842

Hurst, J., Hoffmann, M., Pace, M., Williams, J. P., Thornhill, J., Hamlyn, E., et al. (2015). Immunological biomarkers predict HIV-1 viral rebound after treatment interruption. Nat. Commun. 6:8495.

Imamichi, H., Smith, M., Adelsberger, J. W., Izumi, T., Scrimieri, F., Sherman, B. T., et al. (2020). Defective HIV-1 proviruses produce viral proteins. Proc. Natl. Acad. Sci. U.S.A. 117, 3704-3710. doi: 10.1073/pnas.1917876117

Jain, V., Hartogensis, W., Bacchetti, P., Hunt, P. W., Hatano, H., Sinclair, E., et al. (2013). Antiretroviral therapy initiated within 6 months of HIV infection is associated with lower T-cell activation and smaller HIV reservoir size. J. Infect. Dis. 208, 1202-1211. doi: 10.1093/infdis/jit311

Jiang, C., Lian, X., Gao, C., Sun, X., Einkauf, K. B., Chevalier, J. M., et al. (2020). Distinct viral reservoirs in individuals with spontaneous control of HIV-1. Nature 585, 261-267.

Jordan, A., Defechereux, P., and Verdin, E. (2001). The site of HIV-1 integration in the human genome determines basal transcriptional activity and response to Tat transactivation. EMBO J. 20, 1726-1738. doi: 10.1093/emboj/20.7.1726

Julg, B., Dee, L., Ananworanich, J., Barouch, D. H., Bar, K., Caskey, M., et al. (2019). Recommendations for analytical antiretroviral treatment interruptions in HIV research trials-report of a consensus meeting. Lancet HIV 6, e259-e268.

Kaiser, P., Joos, B., Niederost, B., Weber, R., Gunthard, H. F., and Fischer, M. (2007). Productive human immunodeficiency virus type 1 infection in peripheral blood predominantly takes place in CD4/CD8 double-negative $\mathrm{T}$ lymphocytes. J. Virol. 81, 9693-9706. doi: 10.1128/jvi.00492-07

Kinloch-de Loes, S., Dorrell, L., Yang, H., Hardy, G. A., Yerly, S., Cellerai, C., et al. (2015). Aviremia 10 years postdiscontinuation of antiretroviral therapy initiated during primary human immunodeficiency virus-1 infection and association with Gag-Specific T-cell responses. Open Forum Infect. Dis. 2:ofv144.

Kuo, H. H., Ahmad, R., Lee, G. Q., Gao, C., Chen, H. R., Ouyang, Z., et al. (2018). Anti-apoptotic protein BIRC5 maintains survival of HIV-1-infected CD4+ T cells. Immunity 48, 1183-1194.e5.

Kwon, K. J., Timmons, A. E., Sengupta, S., Simonetti, F. R., Zhang, H., Hoh, R., et al. (2020). Different human resting memory $\mathrm{CD} 4^{+} \mathrm{T}$ cell subsets show similar low inducibility of latent HIV-1 proviruses. Sci. Transl. Med. 12:eaax6795. doi: 10.1126/scitranslmed.aax6795

Lassen, K. G., Bailey, J. R., and Siliciano, R. F. (2004). Analysis of human immunodeficiency virus type 1 transcriptional elongation in resting CD4+ T cells in vivo. J. Virol. 78, 9105-9114. doi: 10.1128/jvi.78.17.9105-9114.2004

Li, J. Z., Etemad, B., Ahmed, H., Aga, E., Bosch, R. J., Mellors, J. W., et al. (2016). The size of the expressed HIV reservoir predicts timing of viral rebound after treatment interruption. AIDS 30, 343-353.

Li, J. Z., Smith, D. M., and Mellors, J. W. (2015). The need for treatment interruption studies and biomarker identification in the search for an HIV cure. AIDS 29, 1429-1432. doi: 10.1097/qad.0000000000000658

Lindqvist, B., Svensson Akusjärvi, S., Sönnerborg, A., Dimitriou, M., and Svensson, J. P. (2020). Chromatin maturation of the HIV-1 provirus in primary resting CD4+ T cells. PLoS Pathog. 16:e1008264. doi: 10.1371/journal.ppat.1008264

Lodi, S., Meyer, L., Kelleher, A. D., Rosinska, M., Ghosn, J., Sannes, M., et al. (2012). Immunovirologic control 24 months after interruption of antiretroviral therapy initiated close to HIV seroconversion. Arch. Intern. Med. 172, 1252-1255. doi: 10.1001/archinternmed.2012.2719

Maenza, J., Tapia, K., Holte, S., Stekler, J. D., Stevens, C. E., Mullins, J. I., et al. (2015). How often does treatment of primary HIV lead to post-treatment control? Antivir. Ther. 20, 855-863. doi: 10.3851/imp2963

Maggiolo, F., Di Filippo, E., Comi, L., and Callegaro, A. (2018). Post-treatment controllers after treatment interruption in chronically HIV-infected patients. AIDS 32, 623-628. doi: 10.1097/qad.0000000000001743

Marini, B., Kertesz-Farkas, A., Ali, H., Lucic, B., Lisek, K., Manganaro, L., et al. (2015). Nuclear architecture dictates HIV-1 integration site selection. Nature 521, 227-231. doi: 10.1038/nature14226

Martin, A. R., Bender, A. M., Hackman, J., Kwon, K. J., Lynch, B. A., Bruno, D., et al. (2020). Similar frequency and inducibility of intact HIV-1 proviruses in blood and lymph nodes. J. Infect. Dis. jiaa736. doi: 10.1093/infdis/jiaa736 [Epub ahead of print].

Martin, G. E., and Frater, J. (2018). Post-treatment and spontaneous HIV control. Curr. Opin. HIV AIDS 13, 402-407. doi: 10.1097/coh.0000000000000488 
Mitchell, J. L., Takata, H., Muir, R., Colby, D. J., Kroon, E., Crowell, T. A., et al. (2020). Plasmacytoid dendritic cells sense HIV replication before detectable viremia following treatment interruption. J. Clin. Invest. 130, 2845-2858. doi: 10.1172/jci130597

Moranguinho, I., and Valente, S. T. (2020). Block-and-lock: new horizons for a cure for HIV-1. Viruses 12:1443. doi: 10.3390/v12121443

Moron-Lopez, S., Telwatte, S., Sarabia, I., Battivelli, E., Montano, M., Macedo, A. B., et al. (2020). Human splice factors contribute to latent HIV infection in primary cell models and blood CD4+ $\mathrm{T}$ cells from ARTtreated individuals. PLoS Pathog. 16:e1009060. doi: 10.1371/journal.ppat.100 9060

Musick, A., Spindler, J., Boritz, E., Pérez, L., Crespo-Vélez, D., Patro, S. C., et al. (2019). HIV infected T cells can proliferate in vivo without inducing expression of the integrated provirus. Front. Microbiol. 10:2204. doi: 10.3389/fmicb.2019. 02204

Namazi, G., Fajnzylber, J. M., Aga, E., Bosch, R. J., Acosta, E. P., Sharaf, R., et al. (2018). The control of HIV after antiretroviral medication pause (CHAMP) study: posttreatment controllers identified from 14 clinical studies. J. Infect. Dis. 218, 1954-1963. doi: 10.1093/infdis/jiy479

Ndung'u, T., McCune, J. M., and Deeks, S. G. (2019). Why and where an HIV cure is needed and how it might be achieved. Nature 576, 397-405. doi: 10.1038/ s41586-019-1841-8

Neidleman, J., Luo, X., Frouard, J., Xie, G., Hsiao, F., Ma, T., et al. (2020). Phenotypic analysis of the unstimulated in vivo HIV CD4 T cell reservoir. Elife. 9:e60933.

Noel, N., Peña, R., David, A., Avettand-Fenoel, V., Erkizia, I., Jimenez, E., et al. (2016). Long-term spontaneous control of HIV-1 is related to low frequency of infected cells and inefficient viral reactivation. J. Virol. 90, 6148-6158. doi: 10.1128/jvi.00419-16

Offersen, R., Yu, W. H., Scully, E. P., Julg, B., Euler, Z., Sadanand, S., et al. (2020). $\mathrm{HIV}$ antibody Fc N-linked glycosylation is associated with viral rebound. Cell Rep. 33:108502. doi: 10.1016/j.celrep.2020.108502

Pankau, M. D., Reeves, D. B., Harkins, E., Ronen, K., Jaoko, W., Mandaliya, K., et al. (2020). Dynamics of HIV DNA reservoir seeding in a cohort of superinfected Kenyan women. PLoS Pathog. 16:e1008286. doi: 10.1371/journal.ppat.10 08286

Pannus, P., Rutsaert, S., De Wit, S., Allard, S. D., Vanham, G., Cole, B., et al. (2020). Rapid viral rebound after analytical treatment interruption in patients with very small HIV reservoir and minimal on-going viral transcription. J. Int. AIDS Soc. 23:e25453.

Pasternak, A. O., Adema, K. W., Bakker, M., Jurriaans, S., Berkhout, B., Cornelissen, M., et al. (2008). Highly sensitive methods based on seminested real-time reverse transcription-PCR for quantitation of human immunodeficiency virus type 1 unspliced and multiply spliced RNA and proviral DNA. J. Clin. Microbiol. 46, 2206-2211. doi: 10.1128/jcm.00 055-08

Pasternak, A. O., and Berkhout, B. (2016). HIV reservoir: finding the right needles in a needlestack. Cell Host Microbe 20, 280-282. doi: 10.1016/j.chom.2016.08. 011

Pasternak, A. O., and Berkhout, B. (2018). What do we measure when we measure cell-associated HIV RNA. Retrovirology 15:13.

Pasternak, A. O., Grijsen, M. L., Wit, F. W., Bakker, M., Jurriaans, S., Prins, J. M., et al. (2020). Cell-associated HIV-1 RNA predicts viral rebound and disease progression after discontinuation of temporary early ART. JCI Insight 5:e134196.

Pasternak, A. O., Lukashov, V. V., and Berkhout, B. (2013). Cell-associated HIV RNA: a dynamic biomarker of viral persistence. Retrovirology 10:41.

Patro, S. C., Brandt, L. D., Bale, M. J., Halvas, E. K., Joseph, K. W., Shao, W., et al. (2019). Combined HIV-1 sequence and integration site analysis informs viral dynamics and allows reconstruction of replicating viral ancestors. Proc. Natl. Acad. Sci. U.S.A. 116, 25891-25899. doi: 10.1073/pnas.19103 34116

Pinzone, M. R., VanBelzen, D. J., Weissman, S., Bertuccio, M. P., Cannon, L., Venanzi-Rullo, E., et al. (2019). Longitudinal HIV sequencing reveals reservoir expression leading to decay which is obscured by clonal expansion. Nat. Commun. 10:728.

Pollack, R. A., Jones, R. B., Pertea, M., Bruner, K. M., Martin, A. R., Thomas, A. S., et al. (2017). Defective HIV-1 proviruses are expressed and can be recognized by cytotoxic T lymphocytes, which shape the proviral landscape. Cell Host Microbe 21, 494-506.e4.

Prator, C. A., Thanh, C., Kumar, S., Pan, T., Peluso, M. J., Bosch, R., et al. (2020). Circulating CD30+CD4+ T cells increase before human immunodeficiency virus rebound after analytical antiretroviral treatment interruption. J. Infect. Dis. 221, 1146-1155. doi: 10.1093/infdis/jiz572

Sáez-Cirión, A., Bacchus, C., Hocqueloux, L., Avettand-Fenoel, V., Girault, I., Lecuroux, C., et al. (2013). Post-treatment HIV-1 controllers with a longterm virological remission after the interruption of early initiated antiretroviral therapy ANRS VISCONTI Study. PLoS Pathog. 9:e1003211. doi: 10.1371/ journal.ppat.100321

Salgado, M., Rabi, S. A., O’Connell, K. A., Buckheit, R. W., Bailey, J. R., Chaudhry, A. A., et al. (2011). Prolonged control of replication-competent dual- tropic human immunodeficiency virus-1 following cessation of highly active antiretroviral therapy. Retrovirology. 8:97. doi: 10.1186/1742-46 90-8-97

Scully, E. P., Gandhi, M., Johnston, R., Hoh, R., Lockhart, A., Dobrowolski, C., et al. (2019). Sex-based differences in human immunodeficiency virus type 1 reservoir activity and residual immune activation. J. Infect. Dis. 219, 1084-1094. doi: 10.1093/infdis/jiy617

Serra-Peinado, C., Grau-Expósito, J., Luque-Ballesteros, L., Astorga-Gamaza, A., Navarro, J., Gallego-Rodriguez, J., et al. (2019). Expression of CD20 after viral reactivation renders HIV-reservoir cells susceptible to Rituximab. Nat. Commun. 10:3705.

Shan, L., Rabi, S. A., Laird, G. M., Eisele, E. E., Zhang, H., Margolick, J. B., et al. (2013). A novel PCR assay for quantification of HIV-1 RNA. J. Virol. 87, 6521-6525. doi: 10.1128/jvi.00006-13

Sharaf, R., Lee, G. Q., Sun, X., Etemad, B., Aboukhater, L. M., Hu, Z., et al. (2018). HIV-1 proviral landscapes distinguish posttreatment controllers from noncontrollers. J. Clin. Invest. 128, 4074-4085. doi: 10.1172/jci120549

Sneller, M. C., Justement, J. S., Gittens, K. R., Petrone, M. E., Clarridge, K. E., Proschan, M. A., et al. (2017). A randomized controlled safety/efficacy trial of therapeutic vaccination in $\mathrm{HIV}$-infected individuals who initiated antiretroviral therapy early in infection. Sci. Transl. Med. 9:eaan8848. doi: 10.1126/ scitranslmed.aan 8848

Steingrover, R., Garcia, E. F., van Valkengoed, I. G., Bekker, V., Bezemer, D., Kroon, F. P., et al. (2010). Transient lowering of the viral set point after temporary antiretroviral therapy of primary HIV type 1 infection. AIDS Res. Hum. Retroviruses 26, 379-387. doi: 10.1089/aid.2009.0041

Stöhr, W., Fidler, S., McClure, M., Weber, J., Cooper, D., Ramjee, G., et al. (2013). Duration of HIV-1 viral suppression on cessation of antiretroviral therapy in primary infection correlates with time on therapy. PLoS One 8:e78287. doi: 10.1371/journal.pone.0078287

Strain, M. C., Little, S. J., Daar, E. S., Havlir, D. V., Gunthard, H. F., Lam, R. Y., et al. (2005). Effect of treatment, during primary infection, on establishment and clearance of cellular reservoirs of HIV-1. J. Infect. Dis. 191, 1410-1418. doi: 10.1086/428777

Strongin, Z., Micci, L., Fromentin, R., Harper, J., McBrien, J., Ryan, E., et al. (2020). Virologic and immunologic features of simian immunodeficiency virus control post-ART interruption in rhesus macaques. J. Virol. 94:e00338-20. doi: 10.1128/JVI.00338-20

Van Gulck, E., Bracke, L., Heyndrickx, L., Coppens, S., Atkinson, D., Merlin, C., et al. (2012). Immune and viral correlates of "secondary viral control" after treatment interruption in chronically HIV-1 infected patients. PLoS One 7:e37792. doi: 10.1371/journal.pone.0037792

Vansant, G., Bruggemans, A., Janssens, J., and Debyser, Z. (2020a). Block-and-lock strategies to cure HIV infection. Viruses 12:84. doi: 10.3390/v12010084

Vansant, G., Chen, H. C., Zorita, E., Trejbalová, K., Miklík, D., Filion, G., et al. (2020b). The chromatin landscape at the HIV-1 provirus integration site determines viral expression. Nucleic Acids Res. 48, 7801-7817. doi: 10.1093/ nar/gkaa536

Veenhuis, R. T., Kwaa, A. K., Garliss, C. C., Latanich, R., Salgado, M., Pohlmeyer, C. W., et al. (2018). Long-term remission despite clonal expansion of replication-competent HIV-1 isolates. JCI Insight 3:e122795.

Violari, A., Cotton, M. F., Kuhn, L., Schramm, D. B., Paximadis, M., Loubser, S., et al. (2019). A child with perinatal HIV infection and long-term sustained virological control following antiretroviral treatment cessation. Nat. Commun. 10:412. 
Vranckx, L. S., Demeulemeester, J., Saleh, S., Boll, A., Vansant, G., Schrijvers, R., et al. (2016). LEDGIN-mediated inhibition of integrase-LEDGF/p75 interaction reduces reactivation of residual latent HIV. EBioMedicine 8, 248-264. doi: 10.1016/j.ebiom.2016.04.039

Wang, Z., Gurule, E. E., Brennan, T. P., Gerold, J. M., Kwon, K. J., Hosmane, N. N., et al. (2018). Expanded cellular clones carrying replication-competent HIV-1 persist, wax, and wane. Proc. Natl. Acad. Sci. U.S.A. 115, E2575-E2584.

Williams, J. P., Hurst, J., Stöhr, W., Robinson, N., Brown, H., Fisher, M., et al. (2014). HIV-1 DNA predicts disease progression and post-treatment virological control. Elife. 3:e03821.

Yukl, S. A., Kaiser, P., Kim, P., Telwatte, S., Joshi, S. K., Vu, M., et al. (2018). HIV latency in isolated patient CD4+ T cells may be due to blocks in HIV transcriptional elongation, completion, and splicing. Sci. Transl. Med. 10:eaa9927.
Zhang, Z. (2012). An in vitro diagnostic multivariate index assay (IVDMIA) for ovarian cancer: harvesting the power of multiple biomarkers. Rev. Obstet. Gynecol. 5, 35-41. doi: 10.2147/cbf.s30228

Conflict of Interest: The authors declare that the research was conducted in the absence of any commercial or financial relationships that could be construed as a potential conflict of interest.

Copyright $\odot 2021$ Pasternak, Psomas and Berkhout. This is an open-access article distributed under the terms of the Creative Commons Attribution License (CC BY). The use, distribution or reproduction in other forums is permitted, provided the original author(s) and the copyright owner(s) are credited and that the original publication in this journal is cited, in accordance with accepted academic practice. No use, distribution or reproduction is permitted which does not comply with these terms. 